\title{
Déictiques et marques énonciatives : fonction(s) référentielle(s) dans Alger, le cri de Samir Toumi
}

Yasmina BAGHBAGHA ${ }^{1}$

\begin{abstract}
This article tries to explore a literary text, Alger le cri of Samir Toumi to analyze enunciative indices. It aims to identify the meanings and social functions of these configurations. We are approaching the structure and discursive processes, as well as the protests of the narrative instance by using the conceptual apparatus of the analysis of the speech. Proposed peculiarities seem important for understanding some messages. We proceed in three stages to identify the referential and thematic functions, which are spatial, temporal and relative to the person. The text shows that the relationship between fictional aesthetics and referential aesthetics leads to patrimonialisation.
\end{abstract}

Keyboard: discourse analysis; deixis; enunciative brands; referential function DOI: $10.24818 / \mathrm{DLG} / 2021 / 38 / 02$

\section{Introduction}

$\mathrm{D}$ ans son article «Écritures algériennes, littéraires algériennes et Histoire (1954-2012) Esquisse d'un panorama », Chaulet-Achour Christiane distingue deux mouvements littéraires algériens. Le premier est constitué par les auteurs à l'écriture engagée dans l'histoire, au sens sartrien du terme, écrivant une littérature indexée à l'histoire, issue de l'expérience in vivo de la domination coloniale. Le second mouvement, composé des auteurs les plus récents, s'organise autour d'une littérature de soi dont l'écriture se veut dégagée de l'histoire, tout en conservant des inscriptions historiques antérieures (conception reprise par Brodziak, 2017 : 2). Depuis le début du XXI ${ }^{\text {e }}$ siècle, le second mouvement qui ambitionne dissocier le roman personnel de l'histoire et de la mémoire collective n'a cessé de prendre de l'ampleur. Samir Toumi est l'un des auteurs qui en font partie.

Né en 1968, à Alger, plus précisément à Bologhine (ex SaintEugène), le romancier passe sa vie entre les études en France et les projets professionnels à Tunis. Il revient à sa ville natale, Alger en 2004. Toumi

${ }^{1}$ Yasmina Baghbagha, Université Aleger 2, Algérie, yyasminebagh@gmail.com

Dialogos • Vol. XXII No. 38/2021 
publie son premier livre Alger, le cri aux éditions Barzakh, en 2013. Dans une quête de soi, de ses origines et de la parole, l'auteur raconte sa vie associée à celle de la capitale algérienne, ville de sa naissance et de son enfance. La fiction tient une empreinte autobiographique à travers les images illustratives ${ }^{2}$ qui accompagnent les descriptions des endroits, des faits et des actions personnels et personnalisés. On y lit dans les premières pages : «je suis né dans un silence à $20 \mathrm{~h}$. Je n'ai pas crié. La sage-femme a vigoureusement secoué mes jambes. Depuis, je cherche le cri ».

Cet article s'inscrit dans la continuité d'une réflexion précédente, intitulée "Mémoire, usages de l'espace et territoire dans Alger, le cri de Samir Toumi », en phase de publication dans Langage et territorialité littéraire en Afrique. Nous avons analysé le récit d'un point de vue sociolinguistique urbain, discipline ayant pour tâche la problématisation de l'espace. Nous nous proposons dans ce qui suit d'approfondir certains aspects sous une nouvelle perspective en utilisant le cadre théorique de l'analyse du discours conçu par Maingueneau "comme discipline qui, au lieu de procéder à une analyse linguistique du texte en lui-même ou à une analyse sociologique ou psychologique de son "contexte", vise à articuler son énonciation sur un certain lieu social» (1996: 11). Nous justifions notre choix théorique par une telle articulation qui prend appui sur le fait social, caractérisant l'analyse du discours. De plus, Bakhtine considère le discours romanesque en tant que phénomène social. " Le roman est une forme proprement compositionnelle des messes verbales. C'est par elle (cette forme) que se réalise dans un objet esthétique, la forme architectonique $^{3} \mathrm{du}$ " couronnement » littéraire d'un événement historique ou social » $(1978: 35)$.

Nous portons un regard critique sur les différentes fonctions référentielles relevées du corpus grâce aux déictiques/marqueurs énonciatifs. Selon Charaudeau et Maingueneau, "on appelle souvent marques ou traces énonciatives les unités linguistiques qui indiquent le renvoi de l'énoncé à son énonciation: pronoms personnels de première et deuxième personne, désinences de verbes, adverbes de temps, adjectifs

${ }^{2}$ Les images illustratives et les cartes postales représentent l'un des procédés qui véhiculent cet aspect autofictionnel. Nous en découvrons d'autres dans ce qui suit.

${ }^{3}$ Elle désigne les valeurs morales et sociales de celui qui produit le texte romanesque, outre les formes esthétiques propres à chaque genre littéraire (Bakhtine, 1978 : 36). 
affectifs... » (2002 : 230). Notre objectif est de détecter les significations et de dégager les thématiques de la trame narrative dans Alger, le cri. Nous problématisons notre champ de travail comme suit : comment se présentent les déictiques/marqueurs spatio-temporels et personnels, dans Alger le cri ? Quelles fonctions et quelles significations assument-ils ? Afin d'y répondre, nous procédons à l'analyse des discours recueillis (du roman) en observant les configurations, spatiales, temporelles et personnelles. Nous terminons par une conclusion qui présente les résultats de notre analyse.

\section{Ancrage théorique}

En nous inscrivant dans le champ de l'analyse du discours, nous faisons appel à la théorie de l'énonciation que nous estimons la mieux appropriée pour notre entreprise. Elle a pour visée la compréhension et l'interprétation des contenus discursifs. Benveniste avance que «c'est une mise en fonctionnement de la langue par un acte individuel d'utilisation » (1974 : 80). Il est question de la conversion de la langue en discours par une réalisation individuelle. Cette théorie s'attache au fonctionnement de la langue dans une situation d'énonciation. Elle étudie les mécanismes de production/interprétation de la parole impliquant tous les éléments linguistiques et extralinguistiques.

En effet, «l'énonciation constitue le pivot de la relation entre la langue et le monde : d'un côté elle permet de représenter dans l'énoncé des faits, mais d'un autre côté elle constitue elle-même un fait, un événement unique défini dans le temps et l'espace "(Charaudeau et Maingueneau, 2002: 228). Dans cette étude, notre démarche consiste à détecter et à analyser les indices énonciatifs. Pour ce faire, nous observons les actes d'énonciation produits par le narrateur/énonciateur. Ils indiquent l'appropriation de la langue par un locuteur afin de construire et de faire passer un message. Comme l'affirme Benveniste, «le locuteur s'approprie l'appareil formel de la langue et il énonce sa position de locuteur par des indices spécifiques d'une part, et au moyen de procédés accessoires de l'autre » $(1970: 14)$.

\section{Analyse du corpus}

Nous avons effectué une relecture approfondie de notre récit. Nous avons sélectionné et relevé les extraits qui répondent à notre problématique 
afin d'en analyser les fonctions référentielles et thématiques personnelle, spatiale et temporelle.

\section{Référence à la personne}

\subsection{Déictique « je »}

L'énonciateur se manifeste à travers « je », il produit son discours et mobilise le système de la langue à son compte. Il s'est servi de plusieurs éléments autobiographiques pour élaborer son œuvre à la première personne. "Dans l'autobiographie, on suppose qu'il y a une identité entre l'auteur d'une part, le narrateur et le protagoniste d'autre part. C'est-à-dire que le « je » renvoie à l'auteur » (Lejeune, $1975: 28$ ).

Dans Alger, le cri, nous remarquons une constance et une association entre le déictique "je» et son référent. Ils renvoient à ce narrateur/énonciateur errant dans les rues, en quête d'une voix : «lorsque je quitte ma terrasse, c'est pour me perdre dans les rues d'Alger, comme dans les rues de ma mémoire. Je retrouve alors mes souvenirs et je délaisse mon présent. Comme ma ville, j'ai oublié mon futur» (ALC: 20), en s'opposant à la conception de Kerbrat-Orecchioni. Selon elle, « ce qui varie avec la situation c'est le référent d'une unité déictique, et non pas son sens, lequel reste constant d'un emploi à l'autre » (1999: 41).

Quant au statut discursif de « je », il désigne l'instance discursive de l'enfant seul et de l'adulte seul. La solitude semble être un choix, puisque l'enfant tient une stratégie comportementale d'hostilité «boudeur, exigeant, cruel » vis-à-vis de son groupe. Alors qu'il n'est pas fils unique pour se sentir seul comme « une brebis galeuse ». Nous lisons à la page 78, du récit: «ma faille est l'enfant seul, je suis l'enfant seul, Alger est cet enfant seul, boudeur, exigeant, cruel, l'enfant terrible de la fratrie, qui doit à tout prix sortir du lot, quitte à devenir la brebis galeuse qu'on stigmatise, l'enfant prêt à tout détruire pour exister ». Cet énoncé est confirmé par un message iconique 4 , une photo datée de 1973 et intitulée «Enfant seul », à la page 76. «Nombreux sont les écrivains qui ont incorporé la photographie dans leur récit de vie, comme une manière non seulement de rendre leurs

${ }^{4}$ L'icône est « un objet qui entretient avec un autre une relation de ressemblance telle qu' on puisse identifier tout de suite dans l'icône on reconnaît le modèle, mis en présence de l'objet on le reconnaît comme celui à qui a servi le modèle à l'icône » (Martinet, 1973 : 61). 
propos véridiques et authentiques, mais surtout d'attester de leur présence dans le monde qu'ils décrivent »(El Nossery, 2014 : 71).

Les deux instances l'enfant seul et l'adulte seul sont imbriquées. La seconde marque sa présence principalement vers la fin du roman. Ainsi, il est question de la progression de la trame romanesque en harmonie avec la progression du narrateur/énonciateur sur un axe temporel. Le récit s'ouvre par le cri de la naissance dans un silence qui renvoie à la solitude « je suis né dans un silence, à 20h, clinique Saint-Eugène, un jour de Mouloud. Je n'ai pas crié » (ALC: 11) et se termine par "l'adulte seul retournera à l'odeur de la terre, au pied de la montagne blessée. Et là, au milieu des oliviers sauvages, il poussa son cri, qui porté par les vents, chevauchera le dos du serpent et glissera le long des rues tortueuses de la ville » (ALC: 164). Les traits de l'enfant persistent et existent chez l'adulte. L'adjectif «seul » est attribué à l'un et à l'autre des deux substantifs, il désigne un même énonciateur.

Par ailleurs, en observant le passage ci-dessus, à la page 164, nous pouvons relever une troisième dimension à ce «je», outre les caractéristiques de l'instance discursive, la mobilité spatiale. Le support scénique du début du récit était la ville « je suis né dans un silence, à 20h, clinique Saint-Eugène...» (ALC: 11), il s'achève sur l'espace de la compagne, à la fin « l'adulte seul retournera à l'odeur de la terre, au pied de la montagne blessée...» (ALC : 164). Synthétisons notre propos à l'aide de la figure 1 .

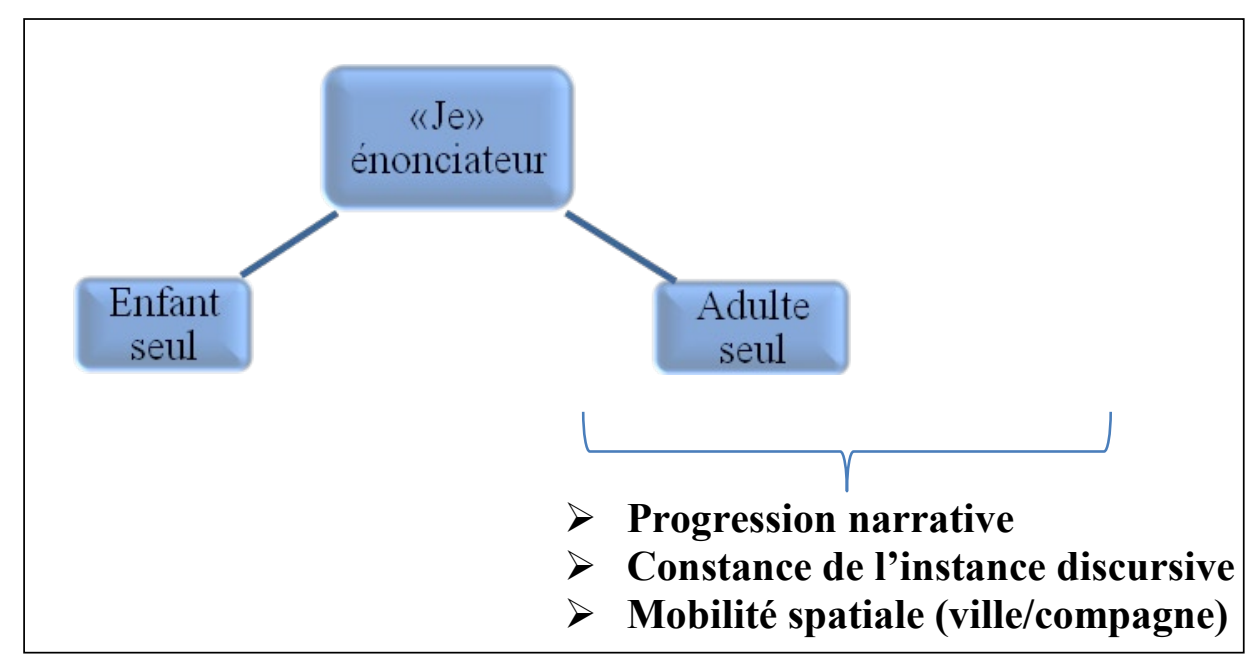

Figure 1. Référence à « je » et ses fonctions 


\subsection{Déictique «elle»}

Les manifestations énonciatives ne se limitent pas au «je» énonciateur du moment que celui-ci suppose un «tu». Ces purs déictiques s'opposent au pronom « il » qui représente la personne dont on parle. La valeur déictique désigne :

Tout un élément linguistique qui dans un énoncé, fait référence à la situation dans laquelle cet énoncé est produit; au moment de l'énoncer (...), au sujet parlant et au participants à la communication. Ainsi les démonstratifs, les adverbes de lieu et de temps, les pronoms personnels, les articles sont des déictiques; ils constituent les aspects indiciels du langage (Dubois et al, 1999 : 132).

Considérons l'énoncé suivant : « je reçois en bourrasque la première bouffée d'air algérois, le cœur se serre un peu plus, je me rapproche d'elle et la faille en moi amorce son réveil » (ALC : 59). L'énonciateur quitte Tunis pour retourner à Alger. Il reprend contact avec sa ville natale dès son arrivée à l'aéroport: "je reçois en bourrasque la première bouffée d'air algérois ». L'accueil semble se faire de manière rude par un brusque coup de vent tourbillonnant.

Alger est désignée par le pronom de la troisième personne «elle », une personne qui dans la communication joue un rôle passif. Cependant, dans ce contexte l'indicateur de la personne "elle» a pu déclencher un malaise refoulé. L'énonciateur ressent un éloignement interne symbolisé par un faussé creusé, «la faille». Autrement dit, le pronom personnel «elle» s'inscrit dans un contraste à la fois externe, par l'acte du rapprochement physique ville-énonciateur lors de l'arrivée de celui-ci à son pays, mais aussi interne qui génère une amertume lors du même contact.

Un peu plus loin, nous lisons: «le cri rend fou. Je croise quotidiennement ces hommes et ces femmes errants qui n'en finissent pas de crier. Ils chuchotent, ils s'énervent, ils hurlent, ils parlent aux fantômes " (ALC: 160). L'énonciateur ne s'identifie pas à « ces hommes et ces femmes", il se contente de les désigner par l'adjectif démonstratif «ces » alors qu'il les « croise quotidiennement ». Il établit une distanciation à leur égard. Ils sont «errants » tout comme lui, mais ils sont capables de crier, contrairement à lui. La succession des verbes de parole renforce l'acte de crier : «chuchoter, hurler, parler» et "s'énerver» aussi, car en s'énervant parfois on crie. À qui adresser cet acte ? «aux fantômes ». Donc, ce sont des actions vaines, c'est pourquoi ces verbes sont précédés du pronom de la troisième personne du pluriel (sujet de ces verbes) exprimant 
la «non-personne». Maingueneau affirme que «le morphème il, à la différence de je-tu est un pro-nom au sens strict, c'est-à-dire un élément anaphorique qui remplace un groupe nominal dont il tire sa référence et qui a été introduit antérieurement dans le discours » (1999: 23).

\subsection{Déictique «tu»}

Après l'analyse des indices personnels, «elle » désignant la ville et « ils » ses occupants, nous nous intéressons à l'actant «tu », à qui renvoie-til ? Il fait référence à la ville, comme le montre l'extrait : "salope de ville, je $\mathbf{t}^{\prime}$ aime ! Chienne de ville! Tu es belle, tu me donne envie de pleurer, je ne pleure pas, un fantôme ne pleure pas » (ALC : 60). La capitale, Alger n'est pas une simple ville. Son image est créée à travers des figures poétiques. L'emploi du pronom personnel «tu » contribue à sa personnification. C'est le fait d'attribuer à une chose abstraite et inanimée les traits, les propriétés d'un être vivant réel, d'une personne. L'énonciateur insulte sa ville «salope » pour les sentiments qu'elle fait naître et auxquels il ne peut résister au point de pleurer. Il lui déclare son amour « je $\mathbf{t}^{\prime}$ aime!", « tu es belle». Le rapport affectif à la ville est marqué par la manifestation simultanée du désir et de la douleur.

C'est dans ce sens qu'il dit: «je pleure nos retrouvailles, nos tête-àtête à venir, toi te réveillant dans ta brume, moi te contemplant de ma terrasse. Je pleure les réveils solitaires dans tes lumières, je pleure tes reflets dans l'eau, ta beauté ta laideur, je pleure sur moi, ou sur toi » (ALC: 61). Telle une femme Alger a un corps, elle tient une posture en "tête-à-tête », elle dispose d'un affect, manifeste des réactions humaines «te réveillant». Elle est à la fois fascinante et répugnante « ta beauté, ta laideur ». Ce point de vue est illustré par Benveniste quand il écrit : « hors du discours affectif, le pronom n'est qu'une forme vide, qui ne peut être attachée ni à un objet ni à un concept » (1966:4).

En somme, la transcendance $\mathrm{du}$ «je » sur «tu» est une relation, « c'est en effet le «je» qui constitue, unilatéralement le «tu», d'après Kerbrat-Orecchioni (1999: 49). L'énonciateur en « je » passe du caractère de la non-personne à travers «elle » et «ils » à une personnification par le tutoiement. Ce qui instaure une certaine pudeur par opposition à l'intimité de se prononcer en «tu », d'une part. Il établit un échange, une interaction entre les deux pôles de l'énonciation je/tu, d'autre part. 


\section{Référence spatiale}

\subsection{Eléments adverbiaux}

L'espace structure le monde narratif et détermine le fonctionnement romanesque des protagonistes et leurs caractéristiques. Dans Alger, le cri, les espaces se concentrent jusqu'à ce qu'ils deviennent des territoires de joie, de souvenirs et d'enfance (Baghbagha, 2021) ${ }^{5}$. Nous nous intéressons dans cette partie aux déictiques spatiaux ayant pour " point de repère $(. .$. la position qu'occupe le corps de l'énonciateur lors de son acte d'énonciation » (Maingueneau, 1999 : 34). Ainsi, dans cet extrait :

De la terrasse, mon regard se plaît à monter et à descendre le long du Chemin Laperlier...J'aime son palmier, au milieu de la rue, juste avant le consulat et sa promesse d'ailleurs, ses petites maisons coloniales perdues au milieu de quelques immeubles défigurés, son coin de compagne en haut du chemin, le parc Tifariti (ALC : 31 )

Nous relevons un adverbe et un ensemble de locutions adverbiales assurant cette fonctionnalité. L'énonciateur procède à la localisation du palmier qui se trouve au Chemin Laperlier grâce aux indices : " au milieu », «avant». Il situe, également les petites maisons coloniales «au milieu» d'autres immeubles, «en haut» $d u$ chemin, le parc Tifariti. Ces microsystèmes sémantiques «correspondent à divers découpages de la catégorie de la spatialité » (Maingueneau, 1999 : 35).

\subsection{Repères toponymiques}

La dimension spatiale se décline en plusieurs toponymes qui participent à l'ancrage référentiel du discours :

Je sortirai pour me perdre dans la foule, je descendrai le boulevard Mohamed V, traverserai la Place Audin pour rejoindre la Grande Poste et suivre la rue Ben M'hidi. Je slalomerai sur les trottoirs, ivres des bruits et de l'agitation, je rejoindrai la Place des Martyrs, puis le Bastion 23 et Padovani (ALC : 101).

Le "je" énonciateur, déambulateur évoque d'autres repères spatiaux :

${ }^{5}$ Que nous avons développé dans «Mémoire, usages de l'espace et territoire dans Alger, le cri de Samir Toumi ». Afin de mieux comprendre notre conception, nous vous invitons à consulter l'article, dès sa publication. 
Je frôle la coupole de Madame l'Afrique, puis je glisse le long de la corniche de Saint-Eugène. Je caresse le Casino en suffoquant dans l'air de la cimenterie de Pointe-Pescade, je vois les rochers dolmen de la plage de Franco, la baraque bleue du Roi de la frite, la maison de Jean Sénac, je vole jusqu'à la villa Tipou (ALC : 103).

Il s'agit des espaces significatifs ayant un ancrage physique et une charge appréciative: "Je me retourne et j'admire la ville, la rampe Chassériau, les arcades des boulevards Zirout Youcef et Che Gueavara, la masse compacte et désordonnée des immeubles, la koubba blanche de Jamaa Ejdid, les arbres du Square Port-Saïd» (ALC : 48). En examinant les passages ci-dessus, nous constatons que le regard porté sur l'espace révèle une organisation thématique : boulevards: Mohamed V, Zirout Youcef et Che Gueavara ; rue Ben M'hidi ; places : Audin, des Martyrs, Square PortSaïd ; édifices: Grande Poste, Bastion 23, Madame l'Afrique, Jamaa Ejdid. Nous avons aussi des espaces à manifestation naturelle, notamment la corniche de Saint-Eugène, plage de Franco entre les deux nous trouvons la cimenterie de Pointe-Pescade.

Tout en s'appuyant sur une cartographie réelle à travers des dénominations toponymiques comme boulevard, rue, place... l'énonciateur fait d'Alger la scène de ses errances. Elle semble être un labyrinthe assurant une représentation spatiale de soi. En effet, la dénomination est une indication de l'espace référentiel dans lequel l'activité énonciative est exercée, l'errance. (Baghbagha, 2018 : 172).

\section{Référence temporelle}

Les indices temporels se présentent sous deux formes, les éléments déictiques et évènementiels que nous analysons.

\subsection{Temps verbal}

Le temps grammatical dominant dans notre corpus est le présent de l'indicatif. Il est lié au moment où l'énonciateur parle, ainsi tout énoncé au présent renvoie à son locuteur :

La forêt de mots m'entoure, sombre, complexe. Je me mets à genoux, je les appelle, tendrement, doucement. Je les vois me guetter, incrédules, attirés, inquiets. Ils se rapprochent, je vois leurs grands yeux luisants, arqués, étonnés, ils se dérobent derrière les arbres, prêts à s'enfoncer dans la forêt (ALC : 75). 
L'étude des affixes temporels confirme qu'il s'agit du présent de deux verbes d'action mettre et appeler, ainsi qu'un verbe de perception voir, conjugués tous avec la première personne du singulier «je»: «Je mets, j'appelle, je vois ». Par ailleurs, ce temps est réservé aux différents référents, également : vent, ville, ciel, mer et souvenirs.

Le vent se fait de plus en plus en plus puissant, la ville lui ordonne de revenir, les éoliennes tournent de fureur, le ciel se charge, la mer se déchaîne, l'Ecosse entre ne Méditerranée. Le vent brouille les lieux et les souvenirs partent en tous sens, impossible de les attraper, ils dansent dans le vent, ils sont inaccessibles (ALC : 88).

Le présent constitue la base du système temporel linguistique. À travers cette démarche, l'énonciateur procède à l'actualisation de son discours afin de créer une certaine affirmation de l'existence et de la réalité des faits. Maingueneau postule que «le point de repère des indications temporelles, c'est le moment où l'énonciateur parle, le «moment d'énonciation », qui définit le présent linguistique » (1999 : 36).

\subsection{Temps évènementiel}

L'événement est un autre aspect qui structure la temporalité romanesque. Alger, le cri reprend différents évènements qui ont marqué l'histoire de l'Algérie: "Alger a toujours connu la guerre. Son histoire, comme la mienne, est fondée par la guerre, les guerres » (ALC : 20) faisant référence à la guerre de libération qui a duré sept ans et demi. Né après l'indépendance, le narrateur/énonciateur a entendu les témoignages des moudjahidine qui ont vécu à cette époque : "Je suis né sous le sceau de la guerre, bercé par les exploits des moudjahidine, au pays du million et demi de chouhada ${ }^{6}$. Allaité par la guerre, je suis l'enfant qui n'a pas crié » (ALC : 34). Nous relevons une « référence cotextuelle " (concept propre à KerbratOrecchioni, 1999 : 41), relative à un élément explicite la guerre, dans le contexte verbal « Je suis né sous le sceau de la guerre».

Deux figures de la scène politique algérienne sont évoquées, Houari Boumediene et Abd El Aziz Bouteflika. Rappelons qu'à l'époque de la publication de ce récit Bouteflika était président, nous pensons que c'est une façon de lui rendre hommage. Observons ce passage. "Chez les vendeurs de rue, le chapelet temporel des cartes postales s'arrête systématiquement à la photographie de Bouteflika jeune, moustache

${ }^{6}$ Le mot est en arabe, il signifie martyrs. 
importante et calvitie précoce, posant à côté du président Boumediene, visage émacié et pommettes saillantes » (ALC : 21). L'énonciateur recourt à la mystification en associant son discours à ces deux figures historiques.

Un autre événement concernant le premier président de l'Algérie indépendante est daté, son décès : «Décembre 1978, Boumediene est mort ! Ce soir-là, je faisais mes devoirs, sous le néon de la seconde cuisine de l'appartement...je me revois peu de temps après sa mort, ce jour où la faille s'est réveillée, en 1980, à El Asnam » (ALC : 22). Un lien est établi entre la mort et le séisme de El Asnam. Le séisme est une métaphore de la faille, représentant un parallèle au séisme intérieur de l'énonciateur. Brodziak précise que « l'écriture de l'histoire devient une vaste entreprise civique à la recherche d'un principe de cohésion capable d'inclure l'expérience individuelle, la mémoire autonome de chacun et de chacune dans le récit collectif de l'Histoire» $(2017: 1)$.

La pluie est tombée à torrents sur Alger, ce jour-là. La boue a déferlé de la montagne, emportant les âmes vers la mer : " en novembre 2001, la boue a charrié les corps vers la mer, créant cette promenade qu'on appelle aujourd'hui l'Esplanade du Millénaire. C'est la mort qui a révélé ces sublimes ouvertures sur la mer, 133 morts, chiffre officiel » (ALC : 105). La capitale a vécu une autre épreuve. Comme nous le constatons, les thématiques évènementielles sont d'ordre politique, social et naturel. Elles fournissent un rôle dans la sauvegarde de l'Histoire et de la mémoire. En effet, « l'alliage entre la mémoire individuelle et la mémoire collective de tous les Algériens tient une configuration dans l'événement, dans l'histoire » (Baghbagha, 2021).

\section{La répétition}

La répétition est un procédé rédactionnel structurant le roman, à travers les différentes manifestations discursives ci-dessus étudiées. Paissa et Druetta en distinguent deux types : la répétition-produit et la répétitionprocessus, l'une se heurte à l'autre parce que la première est résultative, donnée pour acquise et la seconde est processuelle et dynamique ne s'arrêtant pas seulement au dire et à son agencement formel mais comprend aussi et surtout ce que dit l'implicite. « Ainsi, en oscillant entre le statut de figure et celui de stratégie, la répétition se confirme être, avant tout, un comportement langagier, pris dans la triade formée par l'intentionnalité, la causalité et la fatalité » (Paissa et Druetta, 2019 : 12-15). 
Cependant, nous pensons à l'existence d'un rapprochement entre la finalité de la répétition comme objet de construction discursive (aspect formel) et sa construction énonciative (aspect sémantique) dans le texte, dans la mesure où l'errance du narrateur/énonciateur est soumise à la loi de causalité. Ce mouvement est la résultante d'un autre mouvement interne refoulé, d'une mélancolie, d'un mal-être traduit par le comportement de redondance linguistique. Celle-ci a un caractère intentionnel. L'énonciateur est en quête de la voix, de la parole, selon Hagege la répétition est « foncièrement construction de l'oral " (1985: 111). L'idée de l'emboitement des deux types de répétition proposé par Paissa et Druetta est présente à travers les exemples ci-dessus analysés. Considérons la figure 2.

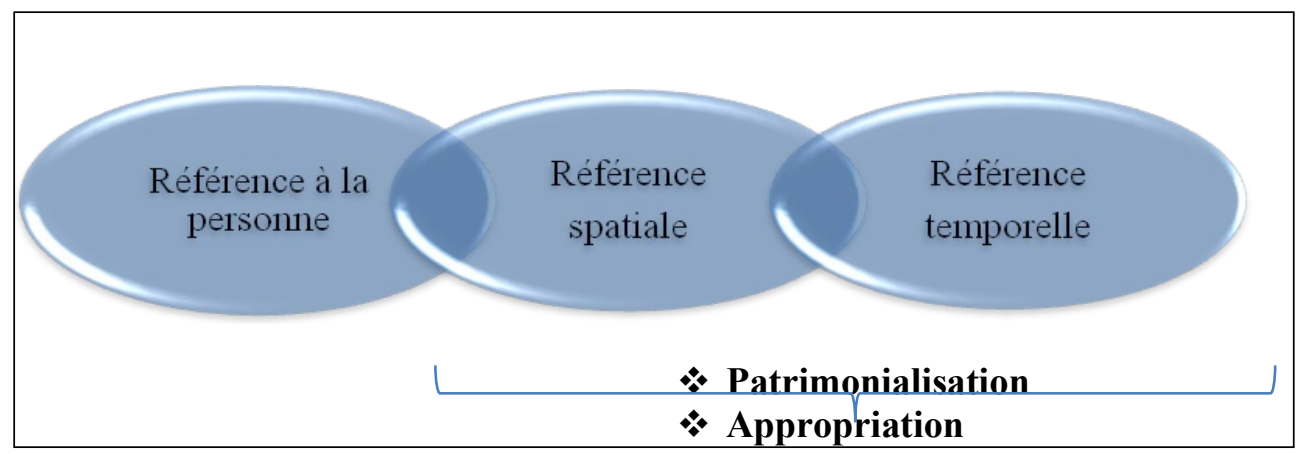

Figure 2. Schéma récapitulatif des fonctions référentielles

\section{Conclusion}

L'analyse des configurations, spatiales, temporelles et personnelles démontre un ensemble de résultats. Le déictique «je » a une incidence sur l'organisation spatiale du roman, voire il structure sa trame narrative. Le mal intérieur psychologique forme un espace puisque le narrateur/énonciateur le sent. Il ne le partage pas avec autrui, ce qui explique l'absence de personnage et la concentration sur soi.

Si la distanciation vis-à-vis de la ville est exprimée par la nonpersonne dans l'emploi du pronom personnel «elle», réveillant la faille, « ils » reflète l'indifférence des occupants de la ville. De plus, le tutoiement instaure une certaine intimité accentuée par des indices de jugement évaluatif et affectif. Ainsi, nous passons de la chosification des personnes par « ils » et la personnification de la ville par « tu».

La référence spatiale se décline sur des éléments, adverbiaux et les repères toponymiques. La dénomination des lieux contribue à définir 
l'identité de l'énonciateur. La construction identitaire passe par le lien à l'espace qui véhicule une charge symbolique de désarroi. Les références temporelles résident dans deux formes, les éléments déictiques portés par la prédominance du présent, en tant que stratégie d'actualisation du discours, et des événements historiques. En effet, la répétition à travers le texte réside à trois niveaux, la reprise des indices linguistiques, le pronom « je », la reprise d'un même énoncé, enfin la reprise en tant que conception structurant le récit.

L'analyse du trio référentiel dévoile deux tendances au patrimoine et à l'appropriation. La patrimonialisation est assurée par des repères matériels, notamment des rues, des places publiques et des séquences événementielles ayant marquées la mémoire collective. Ces composantes sont sujettes d'une appropriation. Il est question d'une appropriation des espaces par les marqueurs linguistiques de possession, en plus d'une appropriation de la langue par les différentes réalisations discursives. Ce que nous pouvons appeler « une appropriation existentielle » (Baghbagha, 2020 : 214). En perspective, nous comptons mener une analyse onomastique des dénominations toponymiques et odonymiques citées par Toumi, dans Alger, le cri.

\section{Bibliographie}

1. BAGHBAGHA, Yasmina (2018), «Déictiques et manifestations identitaires à travers les enseignes commerciales de Ben Aknoun », Cahiers de linguistique, $\mathrm{n}^{\circ} 44$, Paris, L’Harmattan, p. 165-180.

2. BGHBAGHA, Yasmina (2020), «Mise en mots et appropriation de l'espace/langues », Revue Norsud, $\mathrm{n}^{\circ} 16$, Université de Misurata, p. 203217.

3. BAGHBAGHA, Yasmina (2021), "Mémoire, usages de l'espace et territoire dans Alger, le cri de Samir TOUMI », Langages littéraires et territorialités en Afrique, Cameroun, Université de Maroua, 12 pages, à paraître.

4. BAKHTINE, Mikhaïl (1978), Esthétique et théorie du roman [1975], Paris, Gallimard.

5. BENVENISTE, Emile (1966), "Le langage et l'expérience humaine », Problèmes du langage, Gallimard, Paris, pp. 3-13.

6. BENVENISTE, Emile (1970), «L'appareil formel de l'énonciation », Langages 217. 
7. BRODZIAK, Sylvie (2017), « Melancholia Algeriana ou fantômes et jeux de mémoires chez deux écrivains de l'Algérie contemporaine », Babel, n³6. http://journals.openedition.org/babel/5025

8. CHARAUDEAU, Patrick et MAINGUENEAU, Dominique (2002), Dictionnaire d'analyse du discours, Paris, Seuil, $661 \mathrm{p}$.

9. CHAULET-ACHOUR, Christiane (2014), « Écritures algériennes, littéraires algériennes et Histoire (1954-2012) Esquisse d'un panorama ", in Papa Samba Diop et Alain Vuillemin (dir.), Les Littératures en langue française, Histoire, Mythe et création, PUR, http://www.christianeachour.net/

10. DUBOIS, Jean et al (1999), Dictionnaire de linguistique, Paris, Librairie Larousse, $516 \mathrm{p}$.

11. EL NOSSERY, Névine (2014), «L'esthétique du fragment dans l'œuvre photo-textuelle de Leila Sebbar», in Nouvelles Etudes Francophones, Vol 29, $\mathrm{n}^{\circ} 1$. Disponible : https://muse.jhu.edu/article /576331

12. HAGEGE, Claude (1985), L'Homme de paroles, Paris, Fayard, Col. Folio.

13. KERBRAT-ORECCHIONI, Catherine (1999), L'énonciation. De la subjectivité dans la langue, Paris, Armand Colin, 267 p.

14. LEJEUNE, Philippe (1975), Le Pacte autobiographique, Paris, Seuil.

15. MAINGUENEAU, Dominique, (1996) les termes clés de l'analyse du discours, Paris, Seuil, $94 \mathrm{p}$.

16. MARTINET, Jeanne (1973), clefs pour la sémiologie, Ed. Seghers, Paris.

17. MAINGUENEAU, Dominique (1999), L'énonciation en linguistique française, Paris, Hachette, $156 \mathrm{p}$.

18. PAISSA, Paola et DRUETTA, Ruggero (dir.) (2019), La Répétition en discours (Louvain-la-Neuve : Academia, L'Harmattan, Collection Au cœur des textes. 